\title{
INCOMPATIBILITY IN THEOBROMA CACAO
}

\author{
RONALD KNIGHT and H. H. ROGERS \\ West African Cacao Research Institute, Tafo, Gold Coast
}

\section{INCOMPATIBILITY MECHANISMS}

Received 26.iv.54

Gerstel (1950) credits Hughes with first recognising a case of incompatibility governed by multiple alleles exhibiting dominance combined with sporophytic control of the pollen reaction. He states that the hypothesis was given in a thesis on sterility in Crepis foetida. In an account of the system, Hughes and Babcock (1950) have pointed out that their results could not be reconciled with any accepted hypothesis of incompatibility.

Three major hypotheses had previously been put forward. In the first, typified by Nicotiana sanderae, a multiple series of alleles at a single locus with each allele acting independently and gametophytic control of the pollen reaction govern the mechanism. With such a system four intra-sterile groups are sometimes obtained from a cross but all are inter-fertile and will backcross to either parent. Hughes and Babcock with Crepis were able to distinguish among the progeny of a cross four intra-sterile groups but these groups were not fully inter-fertile nor would all of them backcross successfully with their parents. Also obtained in Crepis were reciprocal cross differences which are unknown among the Nicotiana types except where homozygotes have been obtained by an artificial breakdown of incompatibility.

Riley (1936) assumed sporophytic control of the pollen reaction and two pairs of alleles to explain his results with Capsella grandiflora. Hughes and Babcock together with Gerstel (1950), who found a Crepis type system in Parthenium argentatum, accept Riley's hypothesis, and the only similarities with their own data considered by them are those of sporophytic control of the pollen reaction, dominance of alleles and homomorphic structure of the flower.

A third group of flowering plants, all of which are heteromorphic, have sporophytic control of the pollen reaction and only two alleles at any one locus. Modifying alleles may occur at other loci to make up the complete incompatibility system. Comparatively few compatibility groups may be formed with these factors and reciprocal cross differences do not occur, whereas in Crepis seven groups were distinguished among the progeny of a single cross and reciprocal cross differences were found. It was evident to Hughes and Babcock that a new hypothesis would have to be formulated to agree with their experimental findings.

The hypothesis they applied to Crepis has been modified by Crowe (1954) to explain results obtained with Cosmos bipinnatus. In this 
species some of the alleles exhibit dominance in the style, in addition to the pollen, but the dominance sequence is not identical in both style and pollen.

\section{PREVIOUS WORK ON THEOBROMA CACAO}

Incompatibility in cacao has interested research workers wherever the crop has been extensively grown. Over most of these areas the tree is grown from seed but where plant breeding has led to the production of improved selections vegetatively propagated material has begun to replace the seedling trees. As the crop directly depends on successful pollination a knowledge of incompatibility, its cause and incidence, is of major importance.

First experimental results indicating its existence in cacao were published by Pound in 1931. The trees he tested could be divided on their setting capacity into those which were self-compatible and those which were self-incompatible. In the following year he demonstrated that pollen from a self-compatible is effective on any stigma but that pollen from a self-incompatible would only cause setting on a self-compatible. Further reports by Marshall (1933) and Voelcker (1936, 1937) confirmed these earlier findings and illustrated their wide application to the Trinidad population. Cope (1938), however, working with young trees found a range from absolute self-incompatibility to self-compatibility. He was not supported by Ostendorf (1948) who, on the evidence of work at Semarang, found the division between the two classes to be clear cut.

Indications of a change from the self-incompatible to self-compatible state was obtained by Pound (1934) and he suggested that physiological factors could modify the genetic expression of incompatibility at certain seasons of the year. This was termed by Pound conditional self-compatibility. His setting criterion was criticised by Voelcker $(1936,1937)$ who found that a count of sets after six days may give a false impression of genetic compatibility and that a truer picture is obtained after a fourteen-day interval. Voelcker's own results give no evidence of conditional self-compatibility. Data published by other workers (Cope, 1938; Ostendorf, 1948) show that there is a small seasonal fluctuation in setting capacity, but they do not consider there to be a change from incompatibility to compatibility.

Posnette (1945) on testing a population introduced to Trinidad from the headwaters of the Amazon (Pound, 1938) found all the trees to be self-incompatiblf. but to some extent cross compatible. They would also cross successfully with tine self-incompatibles of the cultivated Trinidad population. A further instance of two self-incompatibles being successfully crossed is given by Müntzing (1947) working in Ecuador.

The cytological basis of incompatibility was studied by Cope (1 $133^{8}, 1939$ ). A statistical comparison of pollen tube growth in compatible and incompatible stigmas was insignificant. He traced the tubes into the ovules and found that up to 3 o hours after pollination the male and female gametes behaved indentically in the two types of cross. He suggests that the mechanism causing abscission of the flower depends on the degree of nuclear activity within the ovules. In a sterile cross the rate of division of the endosperm nuclei is much less than in a fertile cross and the abscission mechanism comes into operation.

\section{MATERIAL AND METHODS}

The material used in this study came as seed from Pound's (1938) collection of cacao at Marper Estate, Trinidad. Most of this collection had come from the upper tributaries of the Amazon where the parent trees had been selected for possible resistance to witches' broom disease, Marasmius perniciosus. Posnette, in making the introduction to the Gold Coast in 1944, found all the trees to be selfincompatible. The beans introduced to the Gold Coast were planted at Tafo in 1945 . 
The trees resulting from three pods, designated T.6o, T.63 and T.86, were the first to be tested as they represented combinations of three of Pound's selections $\mathrm{Na} 32, \mathrm{~Pa} 7$ and $\mathrm{Pa} 35$. Thus T.6o was derived from the cross $\mathrm{Pa} 7 \times \mathrm{Na}_{32} ; \mathrm{T} .63$ from $\mathrm{Pa} 35 \times \mathrm{Na} 32$ and $\mathrm{T} .86$ from $\mathrm{Pa} 35 \times \mathrm{Pa} 7$.

Flowers were protected from insect visitation by gauze-covered tubes. Emasculation is unnecessary in cacao. A minimum of five but mainly ten pollinations were made for each cross and were considered successful if the flowers remained on the tree for ten days. The incompatibility reaction is decisive and most of the flowers of an incompatible cross had fallen by the fourth or fifth day.

The 28 trees comprising T.6o were selfed and as many as possible crosses between sister plants were made. T.63 and T.86, each numbering 27 trees, were similarly tested.

TABLE I

\begin{tabular}{|c|c|c|c|c|c|c|c|c|c|c|c|c|}
\hline & $\sigma^{*}$ & A & I & B & $F$ & $\mathrm{Na}_{32}$ & E & J & D & C & G & H \\
\hline q & $\underset{\text { genes }}{\mathbf{S}}$ & $\begin{array}{l}1 \cdot 2 \\
1 \cdot 4\end{array}$ & $\begin{array}{l}1.3 \\
1.5\end{array}$ & $2 \cdot 5$ & $2 \cdot 5$ & $2 \cdot 4$ & $2 \cdot 3$ & 3.5 & 3.4 & 4.5 & 4.5 & 5.5 \\
\hline A & $\begin{array}{l}I \cdot 2 \\
I \cdot 4\end{array}$ & - & - & + & $\ldots$ & + & + & + & + & + & + & + \\
\hline I & $\begin{array}{l}1 \cdot 3 \\
1 \cdot 5\end{array}$ & - & - & $\ldots$ & + & + & $\ldots$ & + & $\ldots$ & $\ldots$ & $\ldots$ & + \\
\hline B & 2.5 & + & + & - & $\ldots$ & - & - & + & + & + & $\ldots$ & + \\
\hline F & $2 \cdot 5$ & + & + & - & - & - & - & + & + & $\ldots$ & + & + \\
\hline $\mathrm{Na} 32$ & $2 \cdot 4$ & $\cdots$ & $\ldots$ & $\cdots$ & $\ldots$ & - & $\cdots$ & $\ldots$ & $\ldots$ & $\ldots$ & $\cdots$ & $\ldots$ \\
\hline E & $2 \cdot 3$ & + & + & - & - & - & - & - & - & + & + & + \\
\hline$J$ & $3 \cdot 5$ & $\cdots$ & + & $\ldots$ & + & + & $\ldots$ & - & - & $\ldots$ & $\ldots$ & + \\
\hline D & 3.4 & + & + & + & + & + & - & - & - & + & $\ldots$ & + \\
\hline C & $4 \cdot 5$ & + & + & + & + & + & + & + & $\ldots$ & - & - & + \\
\hline G & $4 \cdot 5$ & + & + & + & + & + & + & + & + & - & - & + \\
\hline $\mathrm{H}$ & $5 \cdot 5$ & + & $\ldots$ & $\ldots$ & + & + & $\ldots$ & $\cdots$ & $\ldots$ & $\cdots$ & $\ldots$ & - \\
\hline
\end{tabular}

\section{RESULTS}

It was found that the 28 plants of T.6o could be separated into three groups (A, B, C) according to their cross relationships. The three groups were intra-sterile and inter-fertile and differed in the number of plants they contained. Thus group A contained 16 trees, group B, 7 trees and group C, 5 trees. The plants of T.86 were likewise 
separated into three intra-sterile and inter-fertile groups, $\mathrm{H}$, I and J consisting of $9, \mathrm{I} 3$ and 5 trees respectively.

Among the T. 63 trees four groups were found (D, E, F, G) two of which, $E$ and $F$, were cross-sterile and could only be distinguished by their relation to the group D. Group E was cross-sterile and group $F$ was cross-fertile with group $D$. The fourth group, $G$, was completely intra-sterile and inter-fertile. The number of trees in $\mathrm{D}, \mathrm{E}, \mathrm{F}$ and $\mathrm{G}$ was $6,6,6$ and 9 respectively.

On cross pollinating between the ten groups recognised in T.6o, T.63 and T.86 it was found that several were cross-sterile. We have only been able to backcross to $\mathrm{Na} 32$ as the parental clones $\mathrm{Na} 32$, $\mathrm{Pa} 7$ and $\mathrm{Pa} 35$ were not introduced to the Gold Coast until October 195I. The clone $\mathrm{Na} 32$ bore sufficient flowers in 1953 to carry out backcrossing. The results of crossing the ten groups and the backcross are given in table $\mathrm{I}$.

\section{GENETIC HYPOTHESIS}

The following genetic hypothesis is proposed to explain the results :-

I. That success or failure of a cross depends on the diploid constitution of both male and female parent.

2. That in the clones $\mathrm{Pa} 7, \mathrm{~Pa} 35$ and $\mathrm{Na} 32$ five alleles at a single locus are operative.

3. These alleles differ in dominance but two are equal. They follow the sequence :-

$$
\text { S. } \mathrm{I}>2=3>4>5 \text {. }
$$

The sterility gene was previously designated W (Knight and Rogers, 1953).

4. Dominance according to the sequence is exhibited by both male and female organs.

Assuming the clones $\mathrm{Pa} 7, \mathrm{~Pa} 35$ and $\mathrm{Na} 32$ to be of the constitution $\mathrm{S}_{1.5}, \mathrm{~S}_{3.5}$ and $\mathrm{S}_{2.4}$ respectively we have as the cross producing the T.6o trees :-

$$
\mathrm{Si}_{.5} \times \mathrm{S}_{2.4} \text {. }
$$

The offspring of this cross will be :-

$$
\begin{array}{llll}
\mathrm{SI}_{1.2} & \mathrm{~S}_{1.4} & \mathrm{~S}_{2.5} & \mathrm{~S}_{4.5}
\end{array}
$$

but because $S_{I}$ is dominant to $S_{2}$ and $S_{4}$ in the two groups $S_{I .2}$ and $\mathrm{SI}_{\mathrm{I} .4}$ these groups will be indistinguishable in their cross relations. Only three intra-sterile inter-fertile groups were found in the analysis of T.6o with group A containing twice, within experimental variation, as many trees as either of the other groups.

Applying the premises of the hypothesis to T.86 the parents are genetically $\mathrm{S}_{3.5}$ and $\mathrm{S}_{\mathrm{r} .5}$ and will give rise to :-

$$
\begin{array}{llll}
\mathrm{SI}_{1} 3 & \mathrm{~S}_{1.5} & \mathrm{~S}_{3.5} & \mathrm{~S}_{5.5}
\end{array}
$$

Si being dominant to $S_{3}$ and $S_{5}$, the two groups $S_{1.3}$ and $S_{I .5}$ will 
behave identically in a cross and cannot be separated by test pollinations. As with T.6o only 3 intra-sterile inter-fertile groups were found in T.86 with one of the groups (I) larger than the other two.

T.63 was the outcome of the cross between $\mathrm{Pa} 35$ and $\mathrm{Na} 32$. The genes involved according to the hypothesis will be $\mathrm{S}_{3.5}$ and $\mathrm{S}_{2.4}$ giving rise to :-

\section{$\begin{array}{llll}\mathrm{S}_{3.4} & \mathrm{~S}_{2.3} & \mathrm{~S}_{2.5} & \mathrm{~S}_{4.5}\end{array}$}

The groups $\mathrm{S}_{2.3}$ and $\mathrm{S}_{2.5}$ could be distinguished experimentally when crossed with $S_{3.4}$. If this test cross was made $S_{2.3}$ would be incompatible with $S_{3.4}$, having the gene $S_{3}$ in common and equal in action to $S_{2}$, but $S_{2.5}$ would be compatible having no common gene. $S_{4.5}$ would make a fourth distinct group. Four groups were found experimentally in T.63 and agree in their cross relations with those deduced from the hypothesis.

Dominance in the ovary in most cases prevents the separation of the progeny of a cross into the expected four groups. Only when two alleles of equal dominance are associated with weaker alleles, as in T.63, are four groups distinguishable.

When the compatibility between the groups of T.6o and T.86 is considered it is evident from the hypothesis that the largest group in T.6o should not intercross successfully with the largest groups in T.86 but that all the other groups of these two T. numbers should be fully inter-fertile. With the crossing of the groups of T.63 and T.6o it would be expected that $S_{2.3}$ and $S_{2.5}$ of T.63 would fail with $S_{2.5}$ of T.6o and the $\mathrm{S}_{4.5}$ of both would be incompatible. Lastly, crossing between the groups of T.86 and T.63, S3.5 of the former would fail with $\mathrm{S}_{3.4}$ and $\mathrm{S}_{2.3}$ of the latter. All other groups would be fully compatible. On backcrossing to $\mathrm{S}_{2.4}\left(\mathrm{Na}_{32}\right)$ failure would be obtained with $\mathrm{S}_{2.5}$ of T.6o and $\mathrm{S}_{2.3}$ and $\mathrm{S}_{2.5}$ of T.63. The results obtained in the field agree in detail with those deduced from the hypothesis.

\section{CYTOLOGICAL INVESTIGATION}

A cytological investigation was carried out to verify previous findings on cacao. Pollen tube growth in known compatible and incompatible crosses was compared at set intervals from the time of pollination. Ovaries were fixed in chrom-acetic formalin, cleared in $n$-butyl alcohol and embedded in paraffin wax. They were then sectioned at $12 \mu$ and stained in crystal violet following the modified schedule of Smith (1934). The pollen tube studies showed that the rate of pollen tube growth was the same in both sterile and fertile crosses. Tubes could be traced down the stylar canal to the ovules.

Sections through the ovary were identical in both types of cross up to 36 hours after pollination. In sterile and fertile crosses the ovules show a breakdown of one of the synergids and the presence of three endosperm nucleoli. These features are particularly noticeable thirty hours after pollination. In an unpollinated ovary the synergids 
remain intact and there are only two endosperm nucleoli. In both types of cross the male gamete that fuses with the endosperm was easily distinguished within the ovule but the second male gamete, that in other species fuses with the egg, was not detected. Our sections only differed from those of the variety, Trinitario, depicted by Cope (1939), in that we were unable to detect the second male gamete and its fusion with the egg. Since the zygote does not normally divide until at least forty-five days after fertilisation (Cheeseman, 1927), by which time an incompatibly pollinated flower has fallen, fusion of male and egg nucleus in an incompatible cross cannot be assumed. From thirty-six hours until the flower of a sterile cross fell at about four days there was a progressive degeneration in the cytoplasm of the ovary. The ovular nuclei become increasingly difficult to detect.

There can be little doubt that the contents of the pollen tubes are liberated in the ovules of cacao and that failure to set results from a subsequent incompatibility.

\section{DISCUSSION}

Multiple allelic incompatibility must depend for its operation on as many specific reactions as there are distinguishable incompatible groups. More than forty have been detected in some species and it has been suggested that the reaction depends for its specificity on complex polysaccharides or proteins. Analogies have been drawn with the serological antigen-antibody reaction and evidence in its support has been obtained by Lewis (1952). Since the inhibitory reaction of the style, according to Lewis, is preformed and not developed as a result of an incompatible stimulus, a further analogy has been drawn with human blood groups where the $A B$ antigens are naturally occurring.

The antibodies in the serum of one blood group oppose antigens other than the one borne by that group- $B$ group serum contains anti-A antibody-whereas, in the plant's incompatibility reaction, genetically similar groups are opposed and the active substances must be spatially separated. In cacao there is no inhibition in the style and it is suggested that these substances are confined to the cytoplasm of the ovules and pollen. This may be a more primitive condition since it leads, as will be discussed later, to the loss of ovules. It is probable that the incompatibility substances are formed prior to gametogenesis, since they are determined by the diploid constitution of the parent, and this principle may hold for all cases where incompatibility is sporophytically determined.

Autopolyploidy is unlikely to affect the production of " antigens" in pollen when the reaction is sporophytic and the genes exhibit dominance. Tetraploid plants of Parthenium showed the same reactions as their diploid sibs (Gerstel, 1950). There is no indication in our data of competitive interaction or mutual weakening of genes. All the combinations of $\mathrm{S}_{1}$ with $\mathrm{S}_{2}, \mathrm{~S}_{3}, \mathrm{~S}_{4}, \mathrm{~S}_{5}$ behave identically. Whether 
ais absolute dominance would be manifest in a triallelic tetraploid, such as $\mathrm{S}_{1223}$, is of interest in the consideration of "antibody" or " antigen" formation. It might indicate if the "antigen" produced is solely that corresponding to the dominant gene or is at so high a concentration, in diploids, as to mask the "antigen" of the lesser gene. It is known from T.63 that two alleles of equal dominance occur in male and female organs, corresponding with $S_{2}$ and $S_{3}$, without a reduction in the strength of either.

Lewis (1947) found from his work with tetraploid Oenothera that dominance could occur between the genes of diploid pollen but that the genes of the style retained their independent action. He points out that this independence is of natural selective value. In a diploid, where the pollen reaction is gametophytic, dominance in the style permits self-compatibility and inbreeding. If the reaction is sporophytic, as it is in cacao, dominance in both style and pollen does not lead to self-compatibility and in this case independence of the stylar genes is no longer of natural selective importance. Dominance in the style results in a higher compatibility between sibs and prevents the reciprocal cross differences found in Parthenium and Crepis where the genes are independent. Cosmos is apparently intermediate between Crepis and cacao, having some alleles independent in the style that are dominant in the pollen. It has been pointed out (Crowe, I954) that there is some evidence for dominance between the alleles of the style in Parthenium, although this possibility is not mentioned by Gerstel. The raising of compatibility between sibs of cacao lowers the outbreeding efficiency (Bateman, 1953) but conserves generative material where the cacao population is restricted, as it frequently is, in the plant's natural habit.

To achieve incompatibility in cacao there is an enormous loss of flowers which is compensated to some extent by their copious production by self-incompatible as opposed to self-compatible trees. Self-incompatibility does not greatly reduce the production of fruit in Trinitario (Cope, 1938). In other plants the stylar sieve segregates incompatible pollen and leaves the ovules open to future fertilisation. Other instances in the literature of failure subsequent to the pollen tubes reaching the ovules are Gasteria verrucosa (Sears, 1937) and Hemerocallis citrina (Stout and Chandler, 1933). Both are rhizomatous and have many ovules within the ovary. The effect on reproduction of loss of ovules may be lessened by vegetative propagation or, when a pollination is mixed, sufficient ovules may be compatibly pollinated for the fruit to continue development.

An hypothesis similar to the one adopted for cacao could explain the results with Capsella grandiflora (Riley, 1936). A system similar to that in cacao was depicted by Bateman (1952) when he considered from first principles the combination of known incompatibility factors. Genetically cacao resembles Bateman's scheme J. He states that this scheme has a close empirical resemblance to the results obtained in 
Capsella. If this is the true explanation multiple allelic incompatibility together with sporophytic control of the pollen reaction will have been found in the three phylogenetically separated families Sterculiaceae, Compositae and Cruciferae.

The cacao population considered in this paper contains no selfcompatible trees. In a second paper it is hoped to relate this system with that evidently operating in Trinitario, where a proportion of the trees are fully self-compatible, and to discuss the evolution of the two systems.

\section{SUMMARY}

I. Incompatibility among the progeny of three clones of Amazon cacao crossed in all combinations was studied.

2. All the progeny were found to be self-sterile but to a limited extent cross-fertile. On the basis of cross relationships each of the progenies of two crosses was separated into three intra-sterile but inter-fertile groups. A third cross had one distinct intra-sterile interfertile group and three intra-sterile groups that were not fully inter-fertile.

3. A genetic hypothesis was proposed to explain the results. It is based on five alleles that exhibit dominance according to the sequence $S .1>2=3>4>5$. The genes occur at the same locus.

4. A cytological investigation showed that incompatibility occurs after the pollen tubes have penetrated the ovules. In the cacao types considered incompatibility is not achieved without a heavy loss of generative material.

5. The incompatibility reaction of pollen and ovule is sporophytic : it is determined by the diploid constitution of the male and female parent respectively.

6. The incompatibility system in Amazon cacao is discussed in relation to dominant gene action and the "antigen-antibody" theory of incompatibility. It is suggested that the active incompatibility substances are confined to the cytoplasm of ovules and pollen.

Acknowledgment.- The authors wish to express their gratitude to $\mathrm{Dr} \mathrm{D}$. Lewis and $\operatorname{Dr}$ A. F. Posnette for their criticisms, and for reading the manuscript, and Dr A. J. Bateman for discussion of the incompatibility system. This paper is published by permission of the Director of Cacao Research.

\section{REFERENCES}

Bateman, A. J. 1952. Self-incompatibility systems in Angiosperms. Heredity, 6, 285-3 10.

CHeEseman, E. E. 1927. Fertilisation and embryogeny in Theobroma cacao L. Ann. Bot., 4 I, $107-126$.

COPE, F. W. I938. Some factors controlling the yield of young cacao-lil. 8th. Ann. Rep. on Cacao Res. (Trin.), pp. 4-15.

COPE, F. W. I939. Studies in the mechanism of self-incompatibility in cacao-II. 9th Ann. Rep. on Cacao Res. (Trin.), pp. 19-23.

CROWE, L. K. I954. Incompatibility in Cosmos bipinnatus. Heredity (in the press). 
GERSTEL, D. U. 1950. Self-incompatibility studies in Guayule-II. Inheritance. Genetics, 35, 482-506.

HUGHES, M. B., AND BABCOCK, E. B. I950. Self-incompatibility in Crepis fatida L. subsp. rhreadifolia Bieb. Genetics, 35, 570-588.

KNIGHT, R., AND ROGERs, H. H. 1953. Sterility in Theobroma cacao L. Nature, I72, I64.

LEWIS, D. 1947. Competition and dominance of incompatibility alleles in diploid pollen. Heredity, $1,85-108$.

LEWIS, D. 1952. Serological reactions of pollen incompatibility substances. P.R.S., B, $140,127-135$.

marshall, J. 1933. Fertility in cacao. 3rd Ann. Rep. on Cacao Res. (Trin.), p. 34. MÜNTZING, A. 1947. Some observations on the pollination and fruit setting in Ecuador cacao. Hereditas, 33, 397-404.

ostendorf, F. W. 1948. Fertility of cacao. Chron. Nat., 104, 101-105.

POSNETTE, A. F. 1945. Incompatibility in Amazon cacao. Trop. Agric. Trin., 22, $184-187$.

pound, F. J. 1931. Studies of fruitfulness in cacao II. 'ist Ann. Rep. on Cacao Res. (Trin.), pp. 26-28.

pound, F. J. 1938. Cacao and Witchbroom Disease of South America. Port of Spain, Yuille.

RILEY, H. P. 1936. The genetics and physiology of self-sterility in the genus Capsella. Genetics, 21, 24-39.

SEARs, E. R. 1937. Cytological phenomena connected with self-sterility in the flowering plants. Genetics, 22, 130-181.

SMITH, F. H. 1934. The use of picric acid with the Gram stain in plant cytology. Stain Tech., 9, 95-96.

stout, A. B., AND CHANDLER, G. 1933. Pollen-tube behavior in Hemerocallis with special reference to incompatibilities. Bull. Torrey Club, 60, 397-41 7.

voelcker, o. J. 1936. Self-incompatibility in cacao. 6th Ann. Rep. on Cacao Res. (Trin.), pp. 2-5.

vorlcker, o. J. 1937. Self-incompatibility in cacao II. 7th Ann. Rep. on Cacao Res. (Trin.), pp. 2-5. 